\title{
ANÁLISIS DE LA VEGETACIÓN SUCESIONAL EN UN FRAGMENTO DE BOSQUE SECO TROPICAL EN TOLUVIEJO-SUCRE (COLOMBIA)
}

\section{Analysis of sussecional vegetation in a tropical dry forest fragment in Toluviejo- Sucre (Colombia)}

\author{
Dayana Olascuaga-Vargas¹, Jorge Mercado-Gómez² \& Luis Roberto Sanchez-Montaño³
}

Olascuaga-Vargas, D., Mercado-Gómez, J. \& Sánchez-Montaño, L. (2016). Análisis de la vegetación sucesional en un fragmento de bosque seco tropical en Toluviejo-Sucre (Colombia). Colombia Forestal, 19(1), 23-40.

Recepción: 15 de Abril de 2015

\section{RESUMEN}

Se realizó la caracterización estructural y composición florística de la vegetación sucesional en un fragmento de bosque seco tropical en Toluviejo-Sucre (Colombia). Se registraron tres estados de sucesión en los que se determinaron los índices de diversidad y se compararon mediante un análisis de correspondencia sin tendencia. Se registraron 138 especies distribuidas en 111 géneros y 42 familias. La familia Fabaceae presentó mayor riqueza con 21 especies y 18 géneros, seguida de Sapindaceae y Rubiaceae. El hábito mejor representado en términos de riqueza de especies para el matorral (estado temprano) fueron los arbustos (37.6\%); en el borde del bosque (estado intermedio) los árboles (58.8\%) y en el bosque (estado tardío) árboles (67.5\%). El estado intermedio presenta mayor diversidad de especies que el estado tardío. El estrato temprano presentó mayor dominancia debido al alto grado de perturbación antrópica y al proceso de colonización de especies. Según el DCA, las etapas que muestran mayor similitud son el El (estado intermedio) y el ETA (estado tardío), debido a las semejanzas en el clima, condiciones edáficas y factores bióticos. Este estudio aporta conocimientos relevantes para fomentar
Aprobación: 24 de agosto de 2015

proyectos de conservación de áreas, así como para planes de manejo de la vegetación.

Palabras clave: Fabaceae, riqueza, similitud, sucesión.

\section{ABSTRACT}

The successional vegetation of a fragment of tropical dry forest in Toluviejo-Sucre (Colombia) was analyzed based on its structure and floristic composition. Three states of succession were found, in which determined diversity index, then compared using a detrended correspondence analysis. There were 138 species distributed in 111 genera and 42 families. The Fabaceae family showed higher richness with 21 species and 18 genera, followed by Sapindaceae and Rubiaceae. The habit or lifestyle better represented in terms of species richness at the scrub (early stage) were shrubs $(37.6 \%)$, in the edge of the forest (intermediate stage) trees $(58.8 \%)$ and in the forest (late stage) trees $(67.5 \%)$. In terms of diversity the intermediate stage presents highest species diversity than the late stage. The early stage showed higher dominance due to the high degree of human disturbance and colonization of species. According to correspondence analysis (DCA), the states showing the greatest similarity are El and ETA, this is due to the similarities in

Universidad de Sucre, Carrera 28 No. 5-267. Sincelejo, Colombia. dayanavargas296@gmail.com. Autor para correspondencia.

2 Universidad de Sucre, Carrera 28 No. 5-267. Sincelejo, Colombia. jorge.mercado@unisucre.edu.co.

3 Universidad de Pamplona. Pamplona, Colombia. Irsanchez@unipamplona.edu.co. 
climate, soil conditions and biotic factors. This study provides important information for the conservation of areas, as well as plans for vegetation management.
Key words: Fabaceae, richness, similitude, succession.

\section{INTRODUCCIÓN}

El bosque seco Tropical (bs-T) es uno de los ecosistemas con mayor número de endemismos, grupos funcionales y diversidad beta registrada en el neotrópico (García et al., 2014), caracterizados según Sánchez-Azofeifa et al. (2005), por presentar vegetación dominada por árboles de hoja caduca (por lo menos el 50\% de los árboles presentes son de hoja caduca en sequía), temperatura media anual $\geq 25^{\circ} \mathrm{C}$, el rango de precipitación anual oscila entre 700 y 2000 mm y presenta tres o más meses secos al año (precipitación $<100$ mm/mes). En la actualidad, estos ecosistemas representan el $42 \%$ de los biomas secos del mundo con aproximadamente $7000000 \mathrm{~km}^{2}$ en su estado original, es decir un 67\% (Dirzo et al., 2001) de bosque, el cual se encuentra en alto grado de amenaza producto de su larga historia de transformación y degradación antrópica (Arango et al., 2003), hasta tal punto que solo en América Latina el $66 \%$ ha sido destruido (Quesada et al., 2009).

En Colombia Etter et al (2008) registraron una extensión 8882854 ha de bosque seco, de las cuales 367761 ha se encuentra en el Caribe, reflejadas en 202423 de bosque natural y 165 338 de bosques intervenidos para uso agrícola (García et al., 2014). El departamento de Sucre cuenta con 14060 ha de bosque en mosaico, que pueden encontrarse en diferentes etapas o estados sucesionales (García et al., 2014) y cuya diversidad vegetal aún no ha sido estudiada, ya que los estudios realizados en este departamento, y en gran parte del Caribe, se han enfocado principalmente en análisis de diversidad de vegetación (Cuervo et al., 1986; Gentry, 1995; Carbonó \& Garcia, 2010; Repizo \& Devia, 2008; García \& Rivera, 2010; Marulanda et al., 2003), estudios fitosociológicos (Patiño \& Rangel, 2011) y algunas observaciones poblacionales realizadas en Attalea butyracea realizado (Uribe et al., 2001). No obstante, existen escasos estudios que analicen y caractericen la sucesión presente en los fragmentos de bosque seco tropical en el departamento de Sucre.

Estudios referentes al análisis de la sucesión del bosque seco se puede incluir los de Almazán-Nuñez et al. (2012) en el suroeste de México, Hartter et al. (2008) en la península de Yucatán y Kalacska et al. (2004) en el noroeste de Costa Rica, los cuales enfocan sus esfuerzos en la determinación de la composición, diversidad y estructura de plantas en diferentes estados de sucesión comparando las etapas tempranas, intermedias y tardías en el proceso de regeneración; e instituyen el concepto de dominancia de especies en los estados maduros, es decir, encuentran que la estructura de la vegetación en los estados intermedios es mucho más compleja en comparación con los tardíos, a diferencia de la etapa temprana que se caracteriza por la abundancia de plantas pioneras típicas (muchos arbustos, árboles pequeños con hierbas y suelo desnudo en áreas abiertas).

En el presente estudio se expone el primer análisis de los estados de sucesión presentes en un fragmento de bosque en proceso de restauración en Sucre, y tiene como principal objetivo describir la composición florística, riqueza de especies y similitud entre etapas de sucesión, mediante la aplicación de índices ecológicos y análisis multivariados.

\section{MATERIALES Y MÉTODOS}

\section{Área de estudio}

El muestreo se realizó a $1 \mathrm{~km}$ del casco urbano de Toluviejo (norte de Colombia) en un remanente 
de bs-T ubicado a $190 \mathrm{~m}$ de altitud en la vía que conduce a San Onofre, con un eje de coordenadas geográficas entre los $9^{\circ} 28^{\prime} 35.7^{\prime \prime}$ latitud norte y $75^{\circ} 25^{\prime} 19.6^{\prime \prime}$ longitud oeste (Figura 1). El clima de la región se caracteriza como típicamente tropical, con influencia de los vientos Alisios y una temperatura promedio de $28.5^{\circ} \mathrm{C}$ (Aguas et al., 2008).

Al igual que toda la costa Caribe, esta zona se encuentra bajo la influencia de la celda de convergencia tropical del norte, fenómeno que determina la presencia una estación seca con duración aproximada de cuatro meses (abril-julio), la cual registra un $42 \%$ de la precipitación anual y otra estación lluviosa (agosto-diciembre) con un 55\% de precipitación (Aguas et al., 2008).

\section{Evaluación de la vegetación}

De acuerdo a recorridos preliminares se seleccionaron tres comunidades vegetales con diferente estadío sucesional los cuales fueron temprano (ETE), intermedio (EI) y tardío (ETA). En cada comunidad vegetal se estableció un transectos tipo RAP (Gentry, 1982) modificado de 50 x 4m. La modificación consistió en la recolecta y censado de todos los individuos que tengan DAP $\leq 1 \mathrm{~cm}$ sobre un lado del transecto, mientras que en el otro fueron procesados los individuos con DAP $\geq 1 \mathrm{~cm}$, esto con el fin de identificar todos los estratos de crecimiento presentes en la sucesión. A todos los individuos censados se les midió el DAP y la altura aproximada. Los estados de sucesión se delimitaron según
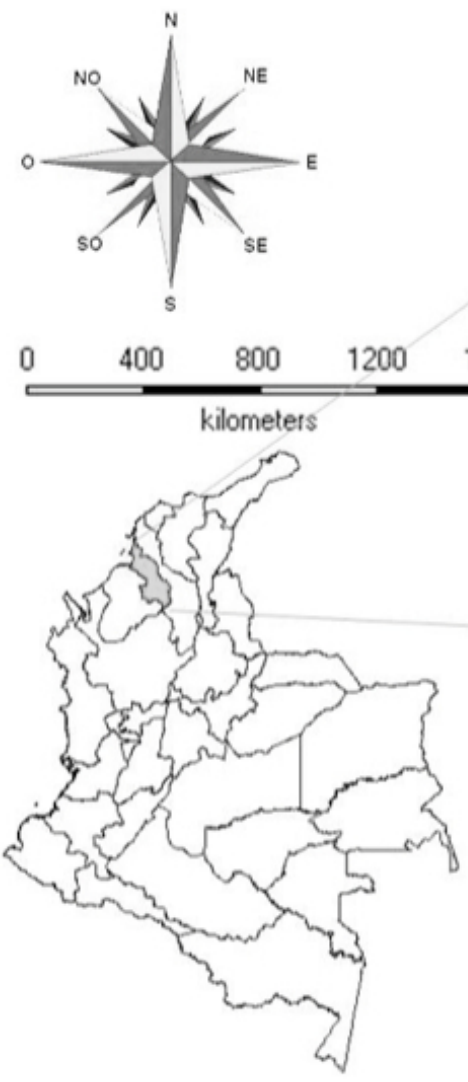

1600
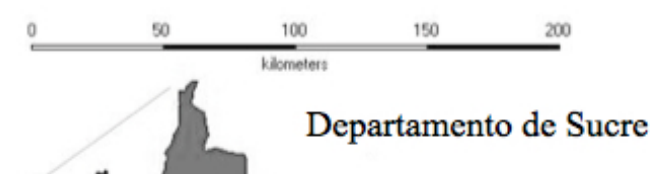

3

Finca la Gaviota

\section{Colombia}

Toluviejo

Figura 1. Ubicación geográfica del área de estudio (Finca la Gaviota) en el municipio de Toluviejo, departamento de Sucre, Colombia. 
Tabla 1. Descripción de los tres estados de sucesión del bosque tropical del sector Santa Rosa (200-300 m de altitud). Adaptado de Arroyo-Mora (2002).

\begin{tabular}{lll}
\hline $\begin{array}{c}\text { Estado } \\
\text { sucesional }\end{array}$ & \multicolumn{1}{c}{ Dosel } & \multicolumn{1}{c}{ Descripción } \\
\hline Temprano & 1 capa, altura media de $6 \mathrm{~m}$ & $\begin{array}{l}\text { Alto porcentaje de árboles de hoja caduca. Muchos arbustos, pequeños } \\
\text { árboles con pastos y suelo desnudo en áreas abiertas. } \\
\text { Capa superior del dosel compuesto por especies de hoja caduca de rápi- } \\
\text { do crecimiento. Segunda capa compuesta por lianas y especies toleran- } \\
\text { tes a la sombra. Presencia de especies de hoja caduca y hoja perenne. } \\
\text { Dosel dominante es de 30 m. Regeneración de especies tolerantes a la } \\
\text { sombra. Pequeña fracción de luz llega al suelo. Dosel superior presenta } \\
\text { superposición de las copas de los árboles de especies de hoja perenne } \\
\text { formando una capa casi continua. }\end{array}$ \\
\hline Tardío & 2 capas, altura media de $10 \mathrm{mas}$ altura media de $30 \mathrm{~m}$ \\
\hline
\end{tabular}

lo descrito por Arroyo-Mora (2002), quien describe tres estados sucesionales en el bosque seco tropical de Santa Rosa-Costa Rica (200-300 m elevación; Tabla 1).

En el proceso de identificación taxonómica con base en el material de referencia (Gentry, 1996), usando el sitio Web (Tropicos-home), se realizaron comparaciones virtuales con colecciones botánicas de referencia del Herbario Nacional Colombiano con la ayuda de expertos botánicos de la Universidad de Sucre. Una vez identificaron los ejemplares, fueron depositados en el en el Herbario de la Universidad de Sucre bajo la enumeración de Olascuaga.

\section{Análisis de la información}

Se evaluó la composición, estructura, abundancia y densidad a nivel de familias y especies para cada transecto y hábito de crecimiento determinando la conformación general del bosque, con lo que se evidenció el cambio en la composición, esto en términos de familias y especies conforme varia la sucesión.

Se realizó un análisis de distribución de clases según la altura y el DAP de las plantas, esto para crear intervalos o categorías y así analizar los estratos verticales en el bosque (Rangel \& Velásquez, 1997). También se calculó el Índice de Valor de Importancia (IVI), para comparar las submuestras dentro de esta misma subunidad paisajística, definiendo qué especies son más representativas para este sitio.

Para calcular la diversidad específica alfa se determinaron los índices de Shannon-Wiener y Simpson (Moreno, 2001), y una vez estimada la diversidad a los datos se les realizó una prueba de normalidad para determinar si estos presentaban una distribución normal y poder realizar el ANOVA. Posterior al análisis de la diversidad alfa, se estimó la diversidad beta por medio del índice de similitud de Jaccard, el cual solo fue realizado a nivel de géneros, ya que en el proceso de identificación taxonómica no fue posible llevar a todos los taxones a nivel de especies. Así mismo, y con el fin de determinar cuáles géneros diferenciaban o generaban similitud entre los puntos muestreados, se llevó a cabo un análisis de correspondencia sin tendencia (DCA), el cual puede generar agrupaciones de taxones con base en la diversidad observada a través de los diferentes estados sucesionales (ICP, 2006). Todos los análisis de diversidad y estadísticos fueron realizados utilizando el programa PAST 2.17 (Hammer et al., 2001).

\section{RESULTADOS}

Riqueza. Un total de 42 familias, 111 géneros y 138 especies fueron identificadas en los tres estados 
sucesionales evaluados (Tabla 2). De estos 138 taxones, 53 fueron identificados hasta género, debido a dos razones principales: carencia de material fértil y colección de referencia para la zona que permitieran realizar identificación por comparaciones. Referente a las familias, Fabaceae fue la que presentó mayor riqueza con 21 especies y 18 géneros, seguida de Sapindaceae y Rubiaceae (Tabla 3).

Tabla 2. Índice de Valor de importancia (IVI) de las especies reportadas en el remanente de bs-T ubicado en ToluviejoColombia: Estadío temprano (ETE). Estadío intermedio (EI). Estadío tardío (ETA). ${ }^{1}$ Especies únicas del matorral. ${ }^{2}$ Especies únicas del borde de bosque. ${ }^{3}$ Especies únicas del bosque. ${ }^{4}$ Especies encontradas en bosque y borde de bosque. ${ }^{5}$ Especies encontradas en borde de bosque y matorral. ${ }^{6}$ Especies encontradas en toda la sucesión. ${ }^{7}$ Especies encontradas en matorral y bosque * Especies no nativas del bs-T.

\begin{tabular}{|c|c|c|c|c|}
\hline \multirow{2}{*}{ Familia } & \multirow{2}{*}{ Especie } & \multicolumn{3}{|c|}{ IVI } \\
\hline & & ETE & EI & ETA \\
\hline Acanthaceae & Justicia sp. ${ }^{4}$ & & 4.1 & 7.4 \\
\hline Acanthaceae & Justicia bracteosa Leonard ${ }^{4}$ & & 7.8 & 5.9 \\
\hline Acanthaceae & Aphelandra alberth-smithii Leonard ${ }^{4}$ & & 8.0 & 4.5 \\
\hline Acanthaceae & Ruellia macrophyllaVahl ${ }^{3}$ & & & 2.3 \\
\hline Anacardiaceae & Astronium graveolens Jacq..$^{5}$ & 95 & 1.6 & \\
\hline Anacardiaceae & Anacardium occidentale L. ${ }^{1}$ & 4.9 & & \\
\hline Anacardiaceae & Astronium fraxinifolium Schott ${ }^{2}$ & & 3.5 & \\
\hline Apocynaceae & Tabernaemontana amygdalifolia Jacq. ${ }^{6}$ & 12.2 & 7.0 & 9.1 \\
\hline Apocynaceae & Tabernaemontana grandiflora (Jacq.) Miers ${ }^{6}$ & 16.5 & 8.2 & 7.0 \\
\hline Apocynaceae & Aspidosperma curranii Standl. ${ }^{3}$ & & & 6.0 \\
\hline Apocynaceae & Rauvolfia viridis Willd. ex Roem. \& Schult. ${ }^{1}$ & 9.6 & & \\
\hline Apocynaceae & Thevetia ahouai (L.) A. DC. ${ }^{1}$ & 2.6 & & \\
\hline Apocynaceae & Asclepias curassavica L. ${ }^{1}$ & 1.2 & & \\
\hline Apocynaceae & Tabernaemontana cymosa Jacq. ${ }^{2}$ & & 2.0 & \\
\hline Apocynaceae & Mandevilla sp. ${ }^{2}$ & & 0.7 & \\
\hline Araceae & Philodendron krugii Engl. ${ }^{2}$ & & 2.6 & \\
\hline Araliaceae & Aralia excelsa (Griseb.) J. Wen² & & 0.7 & \\
\hline Asteraceae & Verbesina sp. ${ }^{1}$ & 4.3 & & \\
\hline Asteraceae & Acmella sp. ${ }^{1}$ & 5.6 & & \\
\hline Bignoniaceae & Tabebuia rosea (Bertol.) DC. ${ }^{3}$ & & & 12.8 \\
\hline Bignoniaceae & Fridericia chica (Bonpl.) L.G. Lohmann ${ }^{4}$ & & 4.0 & 7.6 \\
\hline Bignoniaceae & Memora cladotricha Sandwith ${ }^{4}$ & & 8.0 & 0.7 \\
\hline Bignoniaceae & Clytostoma pterocalyx Sprague ex Urb. ${ }^{3}$ & & & 0.6 \\
\hline Bignoniaceae & Tecoma stans (L.) Juss. ex Kunth ${ }^{1}$ & 9.4 & & \\
\hline Bignoniaceae & Crescentia cujete L. ${ }^{1}$ & 26.1 & & \\
\hline Bignoniaceae & Fridericia conjugata (Vell.) L.G. Lohmann ${ }^{2}$ & & 2.7 & \\
\hline Bignoniaceae & ${\text { Fridericia sp. }{ }^{2}}^{2}$ & & 2.0 & \\
\hline Bixaceae & Cochlospermum orinocense (Kunth) Steud. ${ }^{2}$ & & 1.2 & \\
\hline Boraginaceae & Cordia collococca L. ${ }^{4}$ & & 1.6 & 2.5 \\
\hline Boraginaceae & Tournefortia sp. ${ }^{1}$ & 7.2 & & \\
\hline Boraginaceae & Heliotropium indicum (L.) Druce ${ }^{1}$ & 8.7 & & \\
\hline Boraginaceae & Cordia toqueve Aubl. ${ }^{2}$ & & 1.8 & \\
\hline Bromeliaceae & Aechmea sp. ${ }^{2}$ & & 0.7 & \\
\hline
\end{tabular}




\begin{tabular}{|c|c|c|c|c|}
\hline \multirow{2}{*}{ Familia } & \multirow{2}{*}{ Especie } & \multicolumn{3}{|c|}{ IVI } \\
\hline & & ETE & EI & ETA \\
\hline Burseraceae & Bursera simaruba (L.) Sarg. ${ }^{4}$ & & 20.6 & 15.4 \\
\hline Burseraceae & Protium sp..$^{2}$ & & 1.8 & \\
\hline Capparaceae & Capparidastrum frondosum (Jacq.) Cornejo \& Iltis ${ }^{4}$ & & 19.6 & 12.6 \\
\hline Capparaceae & Capparis indica (L.) Druce ${ }^{6}$ & 1.4 & 2.8 & 0.6 \\
\hline Capparaceae & Morisonia americana L. ${ }^{2}$ & & 2.0 & \\
\hline Capparaceae & Capparis amplissima Lam. $^{2}$ & & 3.1 & \\
\hline Caricaceae & Carica quercifolia (A. St.-Hil.) Hieron. ${ }^{3}$ & & & 1.9 \\
\hline Combretaceae & Terminalia catappa L.² & & 2.0 & \\
\hline Combretaceae & Terminalia amazonia (J.F. Gmel.) Exell ${ }^{2}$ & & 0.7 & \\
\hline Convolvulaceae & Turbina abutiloides (Kunth) O’Donell ${ }^{2}$ & & 2.0 & \\
\hline Costaceae & Costus sp. ${ }^{2}$ & & 0.7 & \\
\hline Cucurbitaceae & Momordica charantia L. ${ }^{5}$ & 2.2 & 0.7 & \\
\hline Cucurbitaceae & Rytidostylis carthaginensis (Jacq.) Kuntze $\mathrm{e}^{5}$ & 1.1 & 1.3 & \\
\hline Elaeocarpaceae & Sloanea $\mathrm{sp} 1^{3}$ & & & 0.6 \\
\hline Elaeocarpaceae & Sloanea sp2 ${ }^{3}$ & & & 0.5 \\
\hline Euphorbiaceae & Acalypha sp. ${ }^{3}$ & & & 16.1 \\
\hline Euphorbiaceae & Hura crepitans L. ${ }^{3}$ & & & 4.2 \\
\hline Euphorbiaceae & Acalypha macrostachya Jacq. ${ }^{3}$ & & & 3.3 \\
\hline Euphorbiaceae & Sapium glandulosum (L.) Morong ${ }^{3}$ & & & 2.3 \\
\hline Euphorbiaceae & Dalechampia scandens L. ${ }^{3}$ & & & 0.5 \\
\hline Euphorbiaceae & Cnidoscolus urens (L.) Arthur ${ }^{1}$ & 14.5 & & \\
\hline Euphorbiaceae & Garcia nutans Vahl' ${ }^{2}$ & & 1.5 & \\
\hline Fabaceae & Acacia sp. $^{3}$ & & & 6.0 \\
\hline Fabaceae & Mucuna mutisiana (Kunth) DC. ${ }^{4}$ & & 2.0 & 4.4 \\
\hline Fabaceae & Desmanthus virgatus L. Willd. ${ }^{4}$ & & 1.3 & 3.8 \\
\hline Fabaceae & Inga sp. ${ }^{3}$ & & & 2.6 \\
\hline Fabaceae & Albizia sp. ${ }^{3}$ & & & 2.3 \\
\hline Fabaceae & Leucaena bolivarensis Britton \& Killip³ & & & 1.7 \\
\hline Fabaceae & Mucuna sp. ${ }^{4}$ & & 2.6 & 1.6 \\
\hline Fabaceae & Bauhinia glabra Jacq. ${ }^{4}$ & & 0.7 & 1.5 \\
\hline Fabaceae & Bauhinia aculeata L. ${ }^{3}$ & & & 1.1 \\
\hline Fabaceae & Nissolia fruticosa Jacq. ${ }^{4}$ & & 1.3 & 1.1 \\
\hline Fabaceae & Crotalaria sp. ${ }^{3}$ & & & 0.6 \\
\hline Fabaceae & Gliricidia sepium (Jacq.) Kunth ex Walp. ${ }^{1}$ & 26.3 & & \\
\hline Fabaceae & Indigofera jamaicensis Spreng. ${ }^{1}$ & 1.2 & & \\
\hline Fabaceae & Desmodium sp. ${ }^{1}$ & 2.2 & & \\
\hline Fabaceae & Enterolobium cyclocarpum (Jacq.) Griseb. ${ }^{2}$ & & 13.1 & \\
\hline Fabaceae & 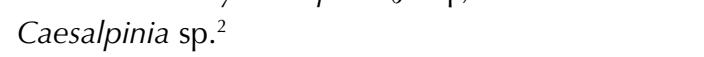 & & 27.9 & \\
\hline Fabaceae & Bauhinia guianensis Aubl.2 & & 2.6 & \\
\hline Fabaceae & Vigna sp. ${ }^{2}$ & & 9.0 & \\
\hline Fabaceae & Machaerium arboreum (Jacq.) Benth² & & 2.0 & \\
\hline Fabaceae & Mimosa pigra L. ${ }^{2}$ & & 0.7 & \\
\hline Fabaceae & $\begin{array}{l}\text { Centrosema sagitatum (Humb. \& Bonpl. ex Willd.) } \\
\text { Brandegee }^{2}\end{array}$ & & 2.6 & \\
\hline Lamiaceae & Ocimum campechiamum Mill. ${ }^{5}$ & 2.2 & 1.3 & \\
\hline Lecythidaceae & Lecythis minor Jacq. ${ }^{5}$ & 9.4 & 23.8 & \\
\hline Malpighiaceae & Stigmaphyllon sp. ${ }^{3}$ & & & 0.5 \\
\hline
\end{tabular}




\begin{tabular}{|c|c|c|c|c|}
\hline \multirow{2}{*}{ Familia } & \multirow{2}{*}{ Especie } & \multicolumn{3}{|c|}{ IVI } \\
\hline & & ETE & EI & ETA \\
\hline Malpighiaceae & Bunchosia pseudonitida Cuatrec. ${ }^{1}$ & 18.8 & & \\
\hline Malvaceae & Cavanillesia platanifolia (Bonpl.) Kunth ${ }^{4}$ & & 13.2 & 18.0 \\
\hline Malvaceae & Malvaviscus sp. ${ }^{3}$ & & & 5.5 \\
\hline Malvaceae & Malvaviscus concinnus Kunth ${ }^{4}$ & & 5.9 & 2.5 \\
\hline Malvaceae & Triumfetta sp. ${ }^{3}$ & & & 1.8 \\
\hline Malvaceae & Guazuma ulmifolia Lam. ${ }^{6}$ & 26.3 & 18.6 & 1.8 \\
\hline Malvaceae & Guazuma tomentosa Kunth ${ }^{4}$ & & 0.9 & 1.3 \\
\hline Malvaceae & Guazuma sp. ${ }^{5}$ & 3.7 & 1.4 & \\
\hline Malvaceae & Sida acuta Burm. f. ${ }^{1}$ & 17.2 & & \\
\hline Meliaceae & Cedrela sp. ${ }^{3}$ & & & 2.3 \\
\hline Meliaceae & Trichilia sp. ${ }^{2}$ & & 0.9 & \\
\hline Moraceae & Brosimum alicastrum Sw. ${ }^{4}$ & & 79.4 & 95.9 \\
\hline Moraceae & Sorocea sp. ${ }^{2}$ & & 1.6 & \\
\hline Moraceae & Maclura tinctoria (L.) D. Don ex Steud. ${ }^{2}$ & & 1.5 & \\
\hline Muntingiaceae & Muntingia calabura L.3 & & & 5.3 \\
\hline Myrtaceae & Eugenia sp. $^{2}$ & & 1.8 & \\
\hline Ochnaceae & Ouratea sp. ${ }^{2}$ & & 1.5 & \\
\hline Olacaceae & Aptandra sp. ${ }^{4}$ & & 4.0 & 2.7 \\
\hline Piperaceae & Piper sp. ${ }^{6}$ & 1.1 & 1.6 & 5.2 \\
\hline Piperaceae & Piper marginatum Jacq. ${ }^{6}$ & 2.2 & 2.7 & 4.4 \\
\hline Poaceae & ${ }^{*}$ Bothriochloa sp. ${ }^{1}$ & 8.9 & & \\
\hline Poaceae & ${ }^{*}$ Brachiaria decumbens Stapf ${ }^{1}$ & 14.6 & & \\
\hline Polygonaceae & Coccoloba sp. ${ }^{2}$ & & 0.9 & \\
\hline Primulaceae & Clavija mezii Pittier ${ }^{4}$ & & 8.5 & 3.6 \\
\hline Ranunculaceae & Clematis haenkeana C. Pres ${ }^{4}$ & & 1.3 & 0.5 \\
\hline Rhamnaceae & ${\text { Gouania sp. }{ }^{2}}^{2}$ & & 0.7 & \\
\hline Rhamnaceae & $\mathrm{sp}^{5}$ & 1.8 & 0.7 & \\
\hline Rubiaceae & Amaioua corymbosa Kunth ${ }^{4}$ & & 23.8 & 46.2 \\
\hline Rubiaceae & Chomelia spinosa Jacq. ${ }^{6}$ & 3.9 & 1.4 & 1.7 \\
\hline Rubiaceae & Hippotis brevipes Spruce ex K. Schum. ${ }^{4}$ & & 3.6 & 1.7 \\
\hline Rubiaceae & Hillia sp. ${ }^{4}$ & & 0.7 & 1.1 \\
\hline Rubiaceae & Morinda citrifolia L. ${ }^{1}$ & 14.6 & & \\
\hline Rubiaceae & Chomelia sp2. ${ }^{1}$ & 8.4 & & \\
\hline Rubiaceae & Chiococca belizensis Lundell ${ }^{2}$ & & 1.4 & \\
\hline Rubiaceae & Psychotria sp. $^{2}$ & & 0.7 & \\
\hline Rubiaceae & Chomelia sp1.2 & & 0.7 & \\
\hline Sapindaceae & Melicoccus bijugatus Jacq. ${ }^{4}$ & & 4.3 & 13.6 \\
\hline Sapindaceae & Melicoccus bijugatus kitan ${ }^{4}$ & & 2.3 & 7.5 \\
\hline Sapindaceae & $\begin{array}{l}\text { Dilodendron costaricense (Radlk.) A.H. Gentry \& } \\
\text { Steyerm. }{ }^{4}\end{array}$ & & 1.4 & 1.2 \\
\hline Sapindaceae & Thinouia sp. ${ }^{4}$ & & 2.6 & 1.1 \\
\hline Sapindaceae & Cardiospermum grandiflorum $\mathrm{Sw}^{3}{ }^{3}$ & & & 1.1 \\
\hline Sapindaceae & Matayba scrobiculata Radlk. ${ }^{3}$ & & & 0.7 \\
\hline Sapindaceae & Serjania sp3. ${ }^{7}$ & 7.2 & & 0.6 \\
\hline Sapindaceae & Paullinia sp. ${ }^{4}$ & & 1.3 & 0.5 \\
\hline Sapindaceae & Allophylus loretensis Standl. Ex J.F. Macbr. ${ }^{3}$ & & & 0.5 \\
\hline Sapindaceae & Serjania communis Cambess. ${ }^{4}$ & & 1.3 & 0.5 \\
\hline
\end{tabular}




\begin{tabular}{|c|c|c|c|c|}
\hline \multirow{2}{*}{ Familia } & \multirow{2}{*}{ Especie } & \multicolumn{3}{|c|}{ IVI } \\
\hline & & ETE & EI & ETA \\
\hline Sapindaceae & Allophylus sp. ${ }^{1}$ & 2.4 & & \\
\hline Sapindaceae & Serjania sp $2{ }^{4}{ }^{4}$ & & 1.3 & 0.5 \\
\hline Sapindaceae & Cardiospermum sp. ${ }^{2}$ & & 1.3 & \\
\hline Sapindaceae & Serjania sp $1 .{ }^{4}$ & & 1.3 & 0.5 \\
\hline Sapotaceae & Chrysophyllum sp. ${ }^{3}$ & & & 0.6 \\
\hline Sapotaceae & Chrysophyllum aff. argenteum Jacq. ${ }^{2}$ & & 1.4 & \\
\hline Sapotaceae & Pouteria sp1.2 & & 0.7 & \\
\hline Sapotaceae & Pouteria sp2. ${ }^{2}$ & & 0.7 & \\
\hline Smilacaceae & Smilax aff. officinalis Kunth $^{5}$ & 9.8 & 4.6 & \\
\hline Urticaceae & Urera baccifera (L.) Gaudich. ex Wedd. ${ }^{4}$ & & 1.2 & 27.0 \\
\hline Urticaceae & Cecropia peltata L. ${ }^{4}$ & & 3.1 & 10.4 \\
\hline Urticaceae & Pouzolzia occidentalis (Liebm.) Wedd. ${ }^{4}$ & & 2.0 & 0.6 \\
\hline Violaceae & Hybanthus sp. ${ }^{1}$ & 5.6 & & \\
\hline
\end{tabular}

Tabla 3. Familias con mayor número de especies y géneros en cada estado de sucesión.

\begin{tabular}{lcccccccccccc}
\hline & \multicolumn{1}{c}{ ETE } & \multicolumn{1}{c}{ EI } & \multicolumn{5}{c}{ ETA } \\
\hline \multirow{2}{*}{ Familia } & \multicolumn{1}{c}{ Especies } & \multicolumn{1}{c}{ Géneros } & \multicolumn{1}{c}{ Especies } & \multicolumn{2}{c}{ Géneros } & Especies & Géneros \\
\cline { 2 - 15 } & $\#$ & $\%$ & $\#$ & $\%$ & $\#$ & $\%$ & $\#$ & $\%$ & $\#$ & $\%$ & $\#$ & $\%$ \\
\hline Fabaceae & 3 & 14.2 & 3 & 14.2 & 12 & 57.1 & 10 & 47.6 & 11 & 52.3 & 9 & 42.8 \\
Sapindaceae & 2 & 14.2 & 2 & 14.2 & 9 & 64.2 & 6 & 42.8 & 12 & 85.7 & 9 & 64.2 \\
Rubiaceae & 3 & 33.3 & 2 & 22.2 & 7 & 77.7 & 6 & 66.6 & 4 & 44.4 & 4 & 44.4 \\
Apocynaceae & 5 & 62.5 & 5 & 62.5 & 4 & 50 & 3 & 37.5 & 3 & 37.5 & 3 & 37.5 \\
Bignoniaceae & 2 & 25 & 2 & 25 & 4 & 50 & 2 & 25 & 4 & 50 & 4 & 50 \\
Malvaceae & 3 & 37.5 & 2 & 25 & 5 & 62.5 & 3 & 37.5 & 6 & 75 & 4 & 50 \\
Euphorbiaceae & 1 & 14.2 & 1 & 14.2 & 1 & 14.2 & 1 & 14.2 & 5 & 71.4 & 4 & 57.1 \\
Capparaceae & 1 & 25 & 1 & 25 & 4 & 100 & 3 & 75 & 2 & 50 & 2 & 50 \\
\hline
\end{tabular}

A nivel de géneros, Brosimum fue el más dominante en toda la sucesión, seguido de Astronium, Capparidastrum, Tabernaemontana, Melicoccus y Amaioua, los cuales presentaron un mayor número de individuos en por lo menos una de las etapas sucesionales. Los géneros restantes están representados algunos por sola una especie $(80.9 \%)$, dos $(13.6 \%)$, tres $(3.6 \%)$ y cuatro especies $(0.9 \%)$ (Figura 2).

Las formas de crecimiento encontradas con mayor frecuencia en los diferentes estados sucesionales fueron: en el matorral (estadío temprano) los arbustos (37.6\%) y las hierbas (31.2\%); en el bosque (estadío tardío) los árboles $(67.5 \%)$ y arbustos $(18.8 \%) ;$ y en el borde del bosque (estadío intermedio) los árboles (58.8\%), arbustos (19.6\%) y lianas (15.4\%) (Figura 3).

Estructura. En términos generales la mayoría de los taxones registrados poseen alturas inferiores a $6 \mathrm{~m}(71.9 \%)$, los que sobresalen presentan entre 14 y 20 m de altura (Tabla 4); el ETA presenta los individuos de mayor porte, mientras que el ETE se caracteriza por presentar individuos en estados juveniles cuya altura no excede los $3.5 \mathrm{~m}$, en cuanto al DAP solo el $3 \%$ supera los $10 \mathrm{~cm}$ y el $97 \%$ restante se encuentra por debajo.

Índice de valor importancia. Las especies con mayor IVI (tabla 2) para el estadío temprano fueron Astronium graveolens, Guazuma ulmifolia y Gliricida sepium. En la etapa intermedia estuvieron 


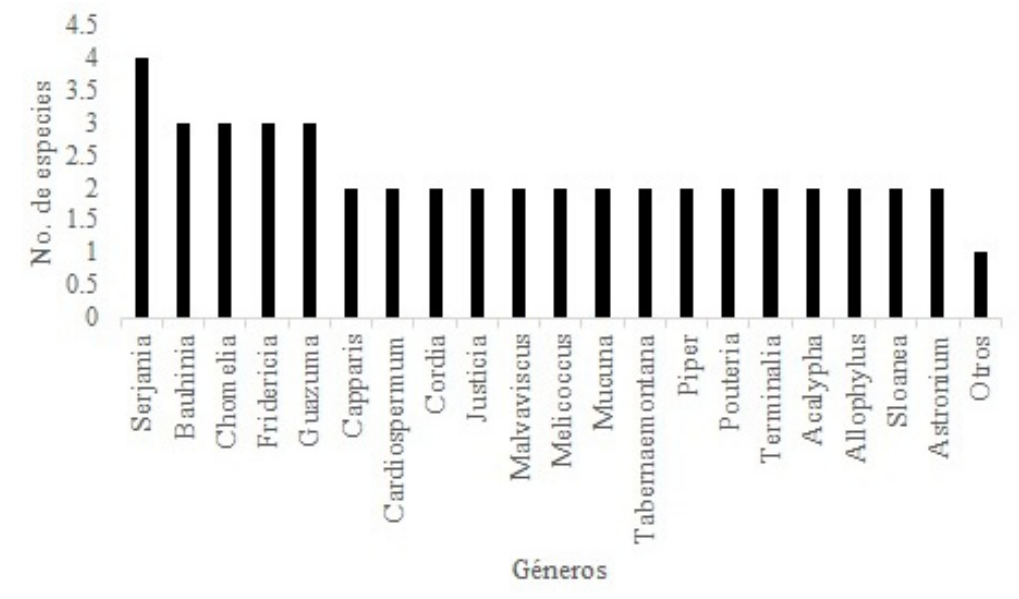

Figura 2. Número de especies reportadas por género en remanente de bosque seco tropical en sucesión (Toluviejo-Colombia).

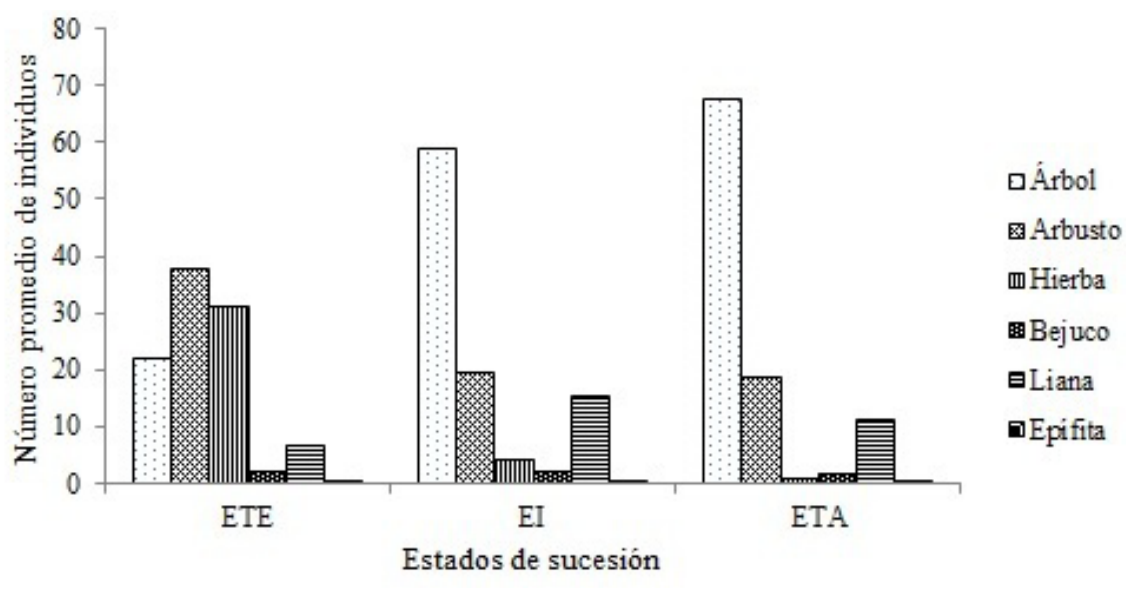

Figura 3. Número promedio de individuos en cada hábito de crecimiento reportado por cada estado de sucesión. Estadío temprano (ETE), estadío intermedio (EI), estadío tardío (ETA).

Tabla 4. Distribución de individuos y especies por clases diamétricas de un remanente de bosque tropical seco en sucesión (Toluviejo-Colombia).

\begin{tabular}{|c|c|c|c|c|c|c|c|c|c|c|c|c|}
\hline \multirow{2}{*}{$\begin{array}{l}\text { Centro de Clase } \\
\text { de DAP }(\mathrm{cm})\end{array}$} & \multicolumn{12}{|c|}{ Centro de Clase de Altura (m) } \\
\hline & $0-2$ & 2.1-4 & 4.1-6 & 6.1-8 & $8.1-10$ & 10.1-12 & 12.1-14 & 14.1-16 & 16.1-18 & 18.1-20 & 20.1-22 & Total \\
\hline $0-2$ & 198 & 110 & 50 & & & & & & & & & \\
\hline $2.1-4$ & 97 & 85 & 24 & 3 & & & & & & & & \\
\hline $4.1-6$ & & 8 & 45 & 62 & 18 & 12 & & & & & & \\
\hline $6.1-8$ & & & & 14 & 12 & 35 & & 10 & & & & \\
\hline $8.1-10$ & & & & & 39 & 20 & & 12 & 3 & & 1 & \\
\hline Total & 295 & 203 & 119 & 79 & 69 & 67 & & 22 & 3 & & 1 & 858 \\
\hline
\end{tabular}


Brosimum alicastrum, Caesalpinia sp. y Lecythis minor. Por último en el estadío tardío se encuentran B. alicastrum, Urera baccifera, Acalypha sp. y Bursera simaruba.

Diversidad alfa. Al realizar la comparación entre la diversidad en los tres estados de sucesión, por medio del ANOVA, con un intervalo de confianza del $95.5 \%$ se estableció que existen diferencias significativas $(p=0.07863)$ entre el número de especies de cada punto muestreado, confirmando la hipótesis que se tenía que los tres sitios poseen desigualdad en su composición florística y en su estructura. Según el estimador Shannon-Wiener, se encontró que el El posee mayor diversidad, seguido del ETA y el ETE. Por otro lado el índice de Simpson arroja resultados en los que se evidencia mayor dominancia de especies en el ETE, seguido del ETA y por último el El (Tabla 5).
Diversidad beta. De acuerdo al índice de similitud de Jaccard, a nivel géneros (Figura 4), se encontró que los estratos que más se asemejan en su composición son El y ETA los cuales comparten 34 especies (Tabla 2) mientras estos dos estadíos con el ET presentan menos relación.

Análisis de correspondencia sin tendencia (DCA): El 85\% de la variación encontrada en los datos es explicada en los dos primeros axis, con un $76 \%$ en el axis 1 y $6 \%$ en el axis 2 (el porcentaje restante se explican en los axis 3 y 4). Sobre el axis 1 se observan 6 agrupaciones que representan los diferentes estados sucesionales y las especies que son compartidas entre cada sucesión (Figura 5). El primer grupo está conformado por los taxones que se distribuyen únicamente en el bosque, en donde es posible distinguir a Tabebuia rosea, Acalypha sp., Aspidosperma curranii, Acalypha macrostachya, Muntingia calabura y Malvaviscus sp. Así mismo,

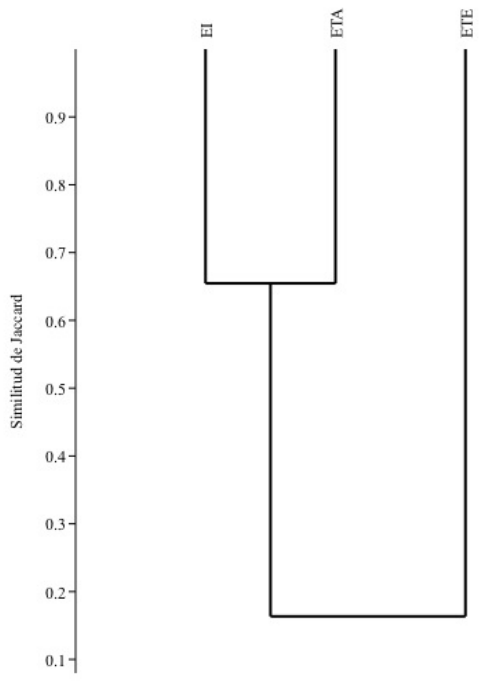

Figura 4. Agrupamiento de similitud por géneros para los sitios muestreados. Matorral (ETE), Borde (EI) y bosque (ETA).

Tabla 5. Índices de diversidad de tres estados de sucesión en un fragmento Bs-t en Toluviejo-Sucre (Colombia). ETE (estado temprano) El (estado intermedio) ETA (estado tardío).

\begin{tabular}{lccc}
\hline & ETE & EI & ETA \\
\hline Simpson & 0.9482 & 0.9735 & 0.9605 \\
Shannon & 3.26 & 4.068 & 3.678 \\
\hline
\end{tabular}


son notables en este grupo algunos taxones como $B$. alicastrum, Amaioua corymbosa, Fridericia chica, Capparidastrum frondosum, Mucuna mutisiana, Melicoccus bijugatus, Urera baccifera, Justicia sp., y Cecropia sp.; no obstante, estos también hacen parte del segundo grupo conformado por aquellas especies que están presentes en el bosque y el borde, pero se encuentran mejor representadas en el bosque. En el segundo grupo también se incluyen a Justicia bracteosa, Desmanthus virgatus, Bursera simaruba, Cordia collococca, Cavanillesia platanifolia, Guazuma tomentosa, Dilodendron costaricense, Pouzolzia occidentalis, Serjania communis, Thinouia sp., Hillia sp. y Paullinia sp., por su parte Aphelandra alberth-smithii, Memora cladotricha, Malvaviscus concinnus, Clavija mezii, Hippotis brevipes, tienen mayor representación en el borde y constituyen el tercer grupo.

En el cuarto grupo se encuentran las especies típicas del borde del bosque, dentro de las cuales se pueden distinguir Tabernaemontana cymosa,
Philodendron krugii, Fridericia conjugata, Turbina abutiloides, Cordia toqueve, Bauhinia guianensis, Centrosema sagitatum, Caesalpinia sp., Fridericia sp., Vigna sp.

El quinto grupo incluye a las especies que normalmente se establecen entre el borde del bosque y el matorral, algunos de ellos son A. graveolens, Momordica charantia, Ocimum campechiamum, Smilax aff. officinalis y Guazuma sp.

El sexto grupo está conformado por los taxones típicos del matorral, dentro de las cuales sobresalen Rauvolfia viridis, Thevetia ahouai, Bunchosia pseudonitida, Cresentia cujete, Cnidoscolus urens, Heliotropium indicum, Sida acuta, Acmella sp., y Verbesina sp.

Finalmente en el séptimo grupo se asocian las especies que poseen una distribución sobre toda la sucesión del bosque Tabernaemontana amygdalifolia, Stemmadenia grandiflora, Piper marginatum, Capparis indica, G. ulmifolia y Chomelia spinosa.

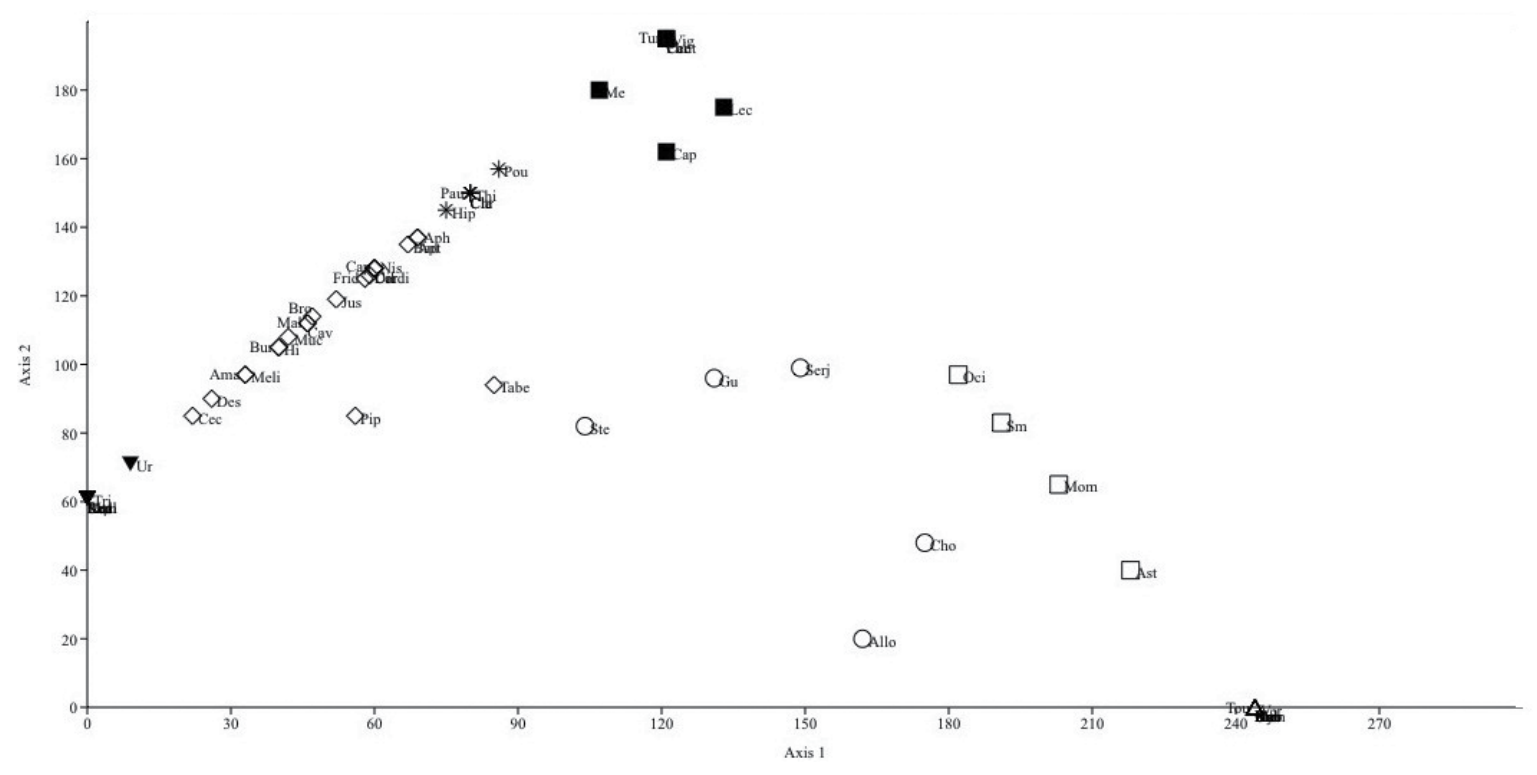

Figura 5. DCA para los géneros encontrados en las etapas sucesionales de un fragmento de Bs-T de ToluviejoSucre (Colombia). $\mathbf{\nabla}$ : géneros representativos del bosque; $\diamond$ : taxones típicos en el bosque aunque con elementos en el borde; *: géneros representativos del borde, aunque con taxones en el bosque; $\mathbf{\square}$ : géneros típicos del borde; $\square$ : géneros típicos del matorral, aunque con elementos en el borde; $\triangle$ : géneros del matorral; $\bigcirc$ : géneros con distribución en toda la sucesión. 


\section{DISCUSIÓN}

La diversidad observada en el remanente de bosque seco de Montes de María en el municipio de Toluviejo (norte de Colombia), muestra una diversidad similar a la detallada en otras localidades que poseen bs-T en el Caribe colombiano, las cuales se encuentran en los departamentos de Atlántico y Bolívar (Rodríguez et al., 2012), en el Magdalena (Marulanda et al., 2003), en la Ilanura Caribe y el valle seco interandino del río Magdalena (Mendoza, 1999), en la isla de Vieja Providencia y Santa Catalina (Ruíz \& Fandiño, 2009).

Al igual que en los estudios mencionados sobre los ecosistemas secos, para Toluviejo la familia Fabaceae presentó la mayor cantidades de especies y abundancia. Este grupo taxonómico es considerado como el más importante en este ecosistema (Linares-Palomino et al., 2011; Pennington et al., 2009), debido a las adaptaciones fisiológicas y estructurales, como son la presencia de hojas compuestas, foliolos pequeños, presencia de espinas o aguijones, hábitos de crecimiento y formas de vida (Ceroni, 2003) y su capacidad de asociarse con microorganismos fijadores de nitrógeno que les permiten crecer en suelos con baja presencia de este elemento.

Así mismo, son importantes en términos de riqueza Apocynaceae, Bignoniaceae, Malvaceae (8), Euphorbiaceae (7) y Capparaceae (4). Esto coincide con lo observado en otras zonas secas del Neotrópico donde las familias con mayor número de especies, además de Fabaceae, son Bignoniaceae, Sapindaceae y Capparaceae en segundo y tercer lugar respectivamente $(\mathrm{IAvH}$, 1998), no obstante, Mendoza (1999) incluye también a Euphorbiaceae y Rubiaceae; mientras que Rodríguez et al. (2012) reportan a Apocynaceae entre las más significativas.

En términos de diversidad según los resultados de los índices, lo detallado en el sitio de muestreo es similar a lo observado en otras localidades con bosques secos tropicales en estado de sucesión; tal es el caso de un fragmento de bosque ubicado al suroeste de México (Almazán-Nuñez et al., 2012), donde los estados tardíos e intermedios presentan mayor riqueza y equitabilidad en comparación con las etapas tempranas, en las cuales existe dominancia de especies; esto se debe al marcado proceso de colonización que inician las especies típicas pioneras del bosque seco. El ETE se caracteriza por ser una zona abierta sin la presencia de individuos de alto porte, con una mayor incidencia de luz, potencial de evaporación y altas temperaturas del aire y del suelo (Del Pozo et al., 1989); además de condiciones ambientales específicas que solo son toleradas por un grupo de taxones como las plantas pioneras. Por su parte las condiciones ambientales del El y el ETA son diferentes a las observadas en el ETE, el dosel encontrado a partir del estadío intermedio genera un microclima propicio para el desarrollo de las especies del bosque maduro, el cual posee mayor complejidad estructural que los dos anteriores.

Con respecto a las formas de crecimiento, se estableció que el patrón en la distribución de los hábitos de crecimiento se asemeja a lo observado en otras localidades del Caribe y los valles interandinos (Rodríguez et al., 2012; Mendoza, 1999). Los arbustos aparecen en toda la sucesión, ubicándose como el segundo tipo de hábito más abundante debido a los factores ecológicos que domina el ecosistema seco, por ejemplo, la defoliación de los árboles dentro del bosque hace que las especies presenten un follaje ralo que facilita una buena iluminación en los estratos inferiores, favoreciendo el desarrollo de los individuos del sotobosque (Carrillo-Fajardo et al., 2007). Así mismo, Badillo (2011) comparte también esta distribución en las formas de crecimiento y lo atribuye a la capacidad que tienen los arbustos de desarrollarse en zonas cuya captación de recursos vitales es débil o fuerte, tal es el caso de los estados temprano y tardío respectivamente.

En el ETE un componente importante fueron los árboles de porte pequeño y arbustos de mediana altura, elementos propios de la vegetación de matorral (García-Hernández \& Jurado 2008). En 
esta etapa se destacan también, hierbas del género Heliotropium, Acmella, Verbesina, Desmodium, Indigofera, Asclepias, y Sida, este hábito es muy común en la fase inicial del proceso de colonización, donde especies pioneras, como las nombradas anteriormente, se dispersan de forma fácil y rápida ampliando su rango de distribución y en la mayoría de las veces facilitando la aparición de especies pioneras de larga duración (Vargas, 2007).

En el El las lianas son un componente primordial, debido a que son una de las primeras formas de vida colonizadoras de claros, especialmente los que son originados por la caída de los árboles, ya que encuentran luz y disponibilidad de soportes para su establecimiento (Bongers et al., 2002), además se presentan con frecuencia en las copas de los árboles durante casi todo el estado tardío de sucesión, esto lo hacen con el fin de alcanzar las zonas más iluminadas en donde se desarrollan y reproducen (Cuevas-Reyes \& Vega-Gutiérrez, 2012). Según Marulanda et al. (2003) estas son un componente importante en los bosques secos en especial aquellos con perturbaciones antrópicas o naturales y a lo largo de los bordes de bosques fragmentados, Ilegando a sobrepasar incluso el número de individuos de árboles.

En la etapa tardía se observa la dominancia de árboles y arbustos, debido al establecimiento de las especies que conforman la estructura del bosque maduro, para este estrato la intensidad del disturbio antrópico es menor al observado en los anteriores, por lo que los individuos tienen un tiempo de regeneración mayor a los demás, presentando alturas superiores a las encontradas en matorral y borde de bosque.

Con respecto a la estructura de la vegetación, la dominancia de árboles de poca altura se debe, según Brown \& Lugo (1990), a que la flora de la sucesión secundaria del Bs-T es menos compleja que la vegetación de este bosque en estado maduro, caracterizándose por tener árboles de pequeño porte con pequeños diámetros, así como una reducida densidad de árboles que superen los $10 \mathrm{~cm}$ de DAP, tal como se observó en Toluviejo.
Además, López et al., (2012) midieron el ritmo del crecimiento diamétrico en los bosques secos tropicales y encuentran que las tasas de crecimiento son muy bajas, varían entre cada especie, pero no superan valores de $3.43 \mathrm{~cm}_{\text {año-1 }}{ }^{-1}$; en promedio los árboles tiene un crecimiento diamétrico que oscila entre 0.49 y $1.06 \mathrm{~cm}_{\text {año }}{ }^{-1}$, mucho más lento que en los bosques húmedos, razón que explica los valores de DAP observados. Esta misma estructura se observa también en otros bosques secos como el Cerro Tasajero de Cúcuta (Carrillo-Fajardo et al., 2007) y el Parque Natural Regional El Vínculo ubicado en el Valle del Cauca (Torres et al., 2012).

Los resultados del Índice de valor importancia en Toluviejo muestran que $A$. graveolens es el taxón dominante en ETE, lo cual se estableció también en el trabajo realizado por Mendoza (1999), en su estudio sobre el bs-T de Forestal Monterrey y Tierra Bomba ubicados en los municipios de Zambrano y Cartagena respectivamente (departamento de Bolívar); por su parte, Los Colorados (Bolívar) y Neguanje (Magdalena) presentaron especies dominantes de árboles de la familia Moraceae, lo cual concuerda con lo observado en este estudio, ya que $B$. alicastrum presentó un alto índice de valor de importancia en El y ETA. Sin embargo, otros autores (Ruíz \& Fandiño, 2009; Marulanda et al., 2003; Yepes \& Villa, 2010; Yepes et al., 2010; Rodríguez et al., 2012) no observan dominancia de individuos pertenecientes a estas familias (Anacardiaceae y Moraceae), probablemente se deba a que el fragmento de bosque estudiado se encuentra en proceso de restauración y $A$. graveolens considerada como especie pionera (Maldonado et al., 2009), debido a que puede soportar condiciones ambientales adversas, por lo que crece rápidamente y se encuentra en los primeros estadíos; por su parte $B$. alicastrum hace parte según Román et al. (2013) de las etapas de sucesión más avanzadas, razón por la que se encontró en los últimos dos estratos.

El agrupamiento de géneros arrojado por el dendrograma con base en el índice de Jaccard, muestra baja similitud entre el ETE en comparación 
con El y ETA, probablemente se deba a que el primero posee mayor intensidad de disturbio, ya que ha sufrido procesos de tala y quema continuos para cultivos de maíz, teniendo menor tiempo de recuperación con respecto a los otros dos estados, manteniéndose así en un proceso de colonización constante. Por su parte, El y ETA no presentan tal alteración y se asemejan en condiciones ambientales y especies, en consecuencia de esto los taxones son diferentes a los hallados en ETE.

DCA. De los resultados obtenidos se pueden distinguir asociaciones que incluyen: plantas del bosque, del borde del bosque, matorral y aquellas especies que se comparten entre los estratos de toda la sucesión, en este sentido, por ejemplo, tenemos un grupo de plantas que hacen parte del matorral y el borde. Este grupo de plantas esta constituidas por especies que en su mayoría son colonizadoras que conforman la primera etapa de sucesión. En esta primera etapa se observó dominancia de pastos y arbustos de tipo pionero hasta intermedio con dosel relativamente bajo (Van Voss et al., 2001) y se encuentra principalmente dominada por individuos con semillas dispersadas por el viento o por la germinación del banco de semillas que posee el suelo, en ocasiones dentro del matorral pueden establecerse las especies clímax del bosque en forma natural; sin embargo, esto depende de la cercanía de fuentes semilleras (Van Voss et al., 2001). Posteriormente con el paso de los años empiezan a aparecer las especies con semillas dispersadas por animales, las cuales corresponderían a etapas siguientes de sucesión como el borde de bosque (Vargas \& Ramírez, 2014). Aquí se encuentran géneros como Tabebuia, Bauhinia, Trichilia y Serjania, que poseen especies pioneras intermedias (Vargas \& Ramírez, 2014), que pueden ser de corta y larga duración. Además, muchos de estos géneros presentan hábitos de crecimiento lianescente lo cual genera en este un de un dosel arbóreo cerrado, sin claros (Guariguata \& Ostertag, 2002).

El grupo de especies compartidas entre borde y bosque incluyen algunos taxones llamados climáticos del Bs-T, es decir, plantas que están mejor adaptadas al lugar y que persisten luego de haber terminado la sucesión (Plana, 2000). Tanto el borde como el bosque poseen condiciones climáticas similares, por ser zonas continuas.. Sin embargo, el borde se caracteriza por ser una etapa de transición entre el matorral y el bosque, razón a la que se atribuye la mayor riqueza de especies, pues presenta individuos tanto de ETE y ETA, esto lo hace diferente al estadío de sucesión tardía. Conell (1978), expone la hipótesis de la perturbación intermedia, en la cual se plantea que la diversidad de una comunidad es alta cuando existen intervenciones en frecuencias e intensidades intermedias, es decir, cuando se está en continuo cambio ya que se retardaría el progreso hacia una condición de estabilidad en la que opere el principio de la exclusión competitiva, lo que indica que existen especies que eliminan a otras, de este modo los cambios ambientales graduales se producen de una manera lo suficientemente alta como para que el proceso de eliminación por competencia rara vez sea completado. Este fenómeno se observó en el estadío intermedio de este estudio, ya que se ve afectado por actividades antrópicas tales como el establecimiento de senderos que permiten el acceso de personas que van en busca de buena madera para trabajos varios, así como el paso en ciertas ocasiones de ganado perteneciente a las fincas aledañas a la zona de estudio.

El bosque, la última etapa de sucesión encontrada en el sitio, se constituye en su mayoría por individuos de larga vida y especies tolerantes a la sombra, con árboles emergentes de gran porte y con presencia de claros de pequeño tamaño que permiten la entrada de luz al sotobosque y la proliferación de arbustos. Las especies más sobresalientes de esta etapa fueron $B$. alicastrum, Bursera simaruba y Cecropia sp. por tener individuos de gran altura.

Por otra parte, los géneros Tabernaemontana, Stemmadenia, Serjania, Guazuma y Chomelia, presentan por lo menos una especie en cada etapa 
sucesional, probablemente se deba a que estos forman parte de la flora habitual de los bosques secos tropicales con algún grado de perturbación, siendo reportadas ya por otros autores (Rodríguez et al., 2012; Mendoza, 1999; Marulanda et al., 2003).

Cabe resaltar que las especies mencionadas en los diferentes grupos son de suma importancia para el comienzo de un proyecto de regeneración del bs-T, ya que la mayoría son nativas, propias de este tipo de vegetación y por tanto existiría menor pérdida en la diversidad del bosque al momento de restaurar y se facilitaría más la recuperación de este tipo de ecosistema, para esta región.

\section{CONCLUSIONES}

El fragmento de bosque seco tropical estudiado se encuentra aún en un estado sucesional temprano, que se caracteriza por tener individuos juveniles de las especies típicas de etapas maduras de sucesión, cuya densidad y área basal son bajas. La diversidad de especies aumenta en los lugares donde existe intervención antrópica moderada pero frecuente, disminuye cuando los disturbios son intensos y altamente repetitivos y tiende a equilibrarse cuando no se tiene ningún tipo de perturbación.

El proceso de regeneración sigue el mismo patrón observado en la sucesión secundaria de otras zonas Neotropicales, en donde primero predominan las hierbas y arbustos (pioneras de corta vida), seguidas de árboles, arbustos y lianas (pioneras de larga vida) y culmina con árboles y arbustos (tolerantes a la sombra) que corresponden el inicio de la comunidad climácica. En la sucesión los estados que presentan mayor similitud en la composición de especies son el estado intermedio y el estado maduro, compartiendo la mayoría de especímenes característicos de la flora tropical del bosque seco. Este estudio permitió incrementar el conocimiento cuantitativo y cualitativo del bosque seco tropical en el departamento de Sucre.

\section{AGRADECIMIENTOS}

A la Universidad de Sucre por la prestación de sus instalaciones. A Carlos Galván por sus valiosos aportes. A Pedro José Álvarez por su colaboración en campo y al Señor Carlos Tamara por su acogida en La Finca La Gaviota.

\section{REFERENCIAS BIBLIOGRÁFICAS}

Aguas, A., Pérez, R., Márquez, C., \& Paternina, R. (2008). Plan de desarrollo de Toluviejo-Sucre. Plan de desarrollo municipal "Oportunidad para Todos". Toluviejo: Alcaldía de Santiago de Tolú-Sucre-Colombia. $220 \mathrm{p}$.

Almazán-Nuñez, R. C., Arizmendi, M., Eguiarte, L., \& Corcuera, P. (2012). Changes in composition, diversity and structure of woody plants in successional stages of tropical dry forest in southwest Mexico. Revista Mexicana de Biodiversidad, 83, 1096-1109.

Arango, N., Armenteras, D., Castro, M., Gottsmann, T., Hernández, O. L., Matallana, C. L., Morales, M., Naranjo, L. G., Renjifo, L. M., Trujillo, A. F., \& Villareal, H. F. (2003). Vacíos de Conservación del Sistema de Parques Nacionales Naturales de Colombia desde una Perspectiva Ecorregional. WWF Colombia, Instituto de investigación de Recursos Biológicos Alexander von Humboldt. Editorial Sepia Ltda. 64 p.

Arroyo-Mora, P. (2002). Forest cover assessment, fragmentation analysis and secondary forest detection for the Chorotega Region, Costa Rica. (Master of Science Thesis). Edmonton University of Alberta CA. 111 p.

Badillo, A. (2011). Fenología de arbustos en distintos estadios de sucesión vegetal en un Bosque Tropical seco. (Trabajo de pregrado, Biología). México: Universidad de Michoacana de San Nicolás de Hidalgo. 74 p.

Bongers, F., Schnitzer, S. A., \& Traore, D. (2002). The importance of lianas and consequences for forest management in West Africa. Bioterre, Rev. Inter. 
Sci. de la Vie et de la Terre. Editions Universitaires de Côte d'Ivoire. N No special, 59-70.

Brown, S., \& Lugo, A. E. (1990). Tropical secondary forest. Journal of Tropical Ecology, 6(1), 1-32.

Carbonó-DeLaHoz, E., \& García, H. (2010). La vegetación terrestre en la ensenada de Neguanje, parque nacional natural Tayrona (Magdalena, Colombia). Caldasia, 32(2), 235-256.

Carrillo-Fajardo, M., Rivera-Díaz, O., \& Sánchez-Montaño, R. (2007). Caracterización florística y estructural del Bosque seco Tropical del Cerro Tasajero, San José de Cúcuta (Norte de Santander), Colombia. Actualidades Biológicas, 29(86), 55-73.

Ceroni, A. (2003). Distribución de las Leguminosas de la parte alta de la Cuenca La Gallega. Morropón. Piura. Ecología Aplicada, 2(1), 9-13.

Conell, J. (1978). Diversity in Tropical Rain Forests and Coral Reefs. Science, New Series. 199(4335), 1302-1310.

Cuervo, A., Barbosa, C., \& De la Ossa, J. (1986). Aspectos ecológicos y etológicos de primates con énfasis en Alouatta seniculus (Cebidae), de la región de Colosó, Serranía de San Jacinto (Sucre) Costa Norte de Colombia. Caldasia, 14, 68-70.

Cuevas-Reyes, P., \&Vega-Gutiérrez, J. (2012). Cambios en la estructura, composición y fenología de plantas epífitas bajo diferentes estadios de sucesión vegetal en un bosque tropical seco. Biológicas, 14(1), 37-44.

Del Pozo, A., Fuentes, E., Hajek, E., \& Molina, J. (1989). Zonación microclimática por efecto de los manchones de arbustos en el matorral de Chile central. Revista Chilena de Historia Natural, 62, 85-94.

Dirzo, R., Young, H. S., Mooney, H. A., \& Ceballos, G. (2001). Seasonally Dry Tropical Forest: Ecology and conservation. Washington: Island Press, Publisher. $408 \mathrm{p}$.

Etter, A., McAlpine, C., \& Possingham, H. (2008). Historical Patterns and Drivers of Landscape change in Colombia since 1500: A regionalized spatial approach. Annals of the Association of American Geographers, 98(1), 2-23.
García-Hernández, J., \& Jurado, E. (2008). Caracterización del Matorral con condiciones pristínas en Linares, N. L., Mexico. Ra Ximhai, 4(1), 1-21.

García, J., \& Rivera, O. (2010). Composición florística del bosque de Agüil (Aguachica, Cesar) con anotaciones sobre su estructura. En J.O. Rangel (ed). Colombia Diversidad Biótica VIII: Media y baja montaña de la serranía de Perijá (pp. 575-601). Instituto de Ciencias Naturales. Bogotá: Editorial Universidad Nacional de Colombia.

García, H., Corzo, G., Isaacs, P., \& Etter, A. (2014). Distribución y estado actual de los remanentes del bioma de Bosque seco tropical en Colombia: Insumos para su gestión. En H. García \& C. Pizano (eds.). El bosque seco tropical en Colombia (pp. 228-251). Instituto de Investigación de Recursos Biológicos Alexander von Humboldt (IAvH). Bogotá: Ediprint Ltda.

Gentry, A. H. (1982). Patterns of Neotropical plant diversity. Evolutionary Biology, 15, 1-84.

Gentry, A. H. (1995). Diversity and floristic composition of neotropical dry forests. En S. H. Bullock, H. A. Mooney \& E. Medina. (eds.). Tropical deciduous Forest Ecosystem (pp. 146-194) Cambridge: University Press Publisher.

Gentry, A. H. (1996). A field guide to the families and genera of woody plants of northwest South America (Colombia, Ecuador, Perú) with supplementary notes on herbaceous taxa. Chicago: University of Chicago Press. 918 p.

Guariguata, M., \& Ostertag, R. (2002). Sucesión secundaria. En M.R. Guariguata \& G.H Kattan. (eds.). Ecología y conservación de bosques neotropicales (pp. 591-623). Costa Rica: Editorial Tecnológica, Cartago.

Hammer, Ø., Harper, DAT \& Ryan, PD. (2001). PAST: Paleontological Statistics Software Package for Education and Data Analysis. Palaeontología Electrónica 4, 1-9.

Hartter, J., Lucas, C., Gaughan, A., \& Lizama, L. (2008). Detecting tropical dry forest succession in a shifting cultivation mosaic of the Yucatán Peninsula, Mexico. Applied Geography, 28, 134-149. 
Instituto de Investigación de Recursos Biológicos Alexander Von Humboldt -IAvH-. (1998). El Bosque seco Tropical (Bs-T) en Colombia. En M. Chavez \& N. A. (eds.). Informe Nacional sobre el estado de la biodiversidad de Colombia, 1997. Bogotá, D.C: Ministerio del Medio Ambiente-Naciones Unidas.

ICP, FOREST. (2006). Estado de los bosques en Europa. Informe Ejecutivo. Programa de Cooperación Internacional para la Evaluación y Seguimiento de los Efectos de la Contaminación Atmosférica en los Bosques. $32 \mathrm{p}$.

Kalacska, M., Sánchez-Azofeifa, G. A., Calvo-Alvarado, J. C., Quesada, M., Rivard, B., \& Hanzan, D. H. (2004). Species composition, similarity and diversity in three successional stages of a seasonally dry tropical forest. Forest Ecology and Management, 200, 227-247.

Linares-Palomino, R., Oliveira-Filho, A. T., \& Pennington, R. T. (2011). Neotropical Seasonally Dry Forests: Diversity, Endemism, and Biogeography of Woody Plants. En R. Dirzo, H. S. Young, H. A. Mooney \& G. Ceballos (eds.). Seasonally Dry Tropical Forests: Ecology and Conservation (pp. 3-21). Island Press/Center for Resource Economics.

López, L., Villalba, R., \& Peña-Claros, M. (2012). Ritmos de crecimiento diamétrico en los bosques secos tropicales: aportes al manejo sostenible de los bosques de la provincia biogeográfica del Cerrado Boliviano. Bosque (Valdivia), 33(2), 211-219.

Maldonado, M., Rodríguez, D. H., Guízar, E., Velásquez, J., \& Nañez, S. (2009). Reducción en riqueza de especies arbóreas por incendios en la Reserva Selva El Ocote, Chiapas. Ciencia forestal en México, 34(106), 127-148.

Marulanda, L.O., Uribe, A., Velásquez, P., Montoya, M.A., Idárraga, A., López, M.C., \& López, J.M. (2003). Estructura y composición de la vegetación de un fragmento de bosque seco en San Sabastián, Magdalena (Colombia). I. Composición de plantas vasculares. Actualidades Biológicas, 25, 17-30.

Mendoza-C., H. (1999). Estructura y riqueza florística del bosque seco tropical en la región Caribe y el valle del río Magdalena, Colombia. Caldasia, 21(1), 70-94.
Moreno, C. E. (2001). Métodos para medir la biodiversidad. M\&T-Manuales y Tesis SEA (Vol. 1). Zaragoza: Editorial UNESCO \& SEA. 84 p.

Patiño, R., \& Rangel, O. (2011). Estudio de caso. La vegetación boscosa en la estación de primates Coloso-Sucre. En O. Rangel-Ch (ed.). Colombia diversidad biótica XI. Patrones de la estructura y de la riqueza de la vegetación en Colombia (pp. 253268). Bogotá: Universidad Nacional de Colombia (Sede Bogotá) Facultad de Ciencias, Instituto de Ciencias Naturales.

Pennington, R. T., Lavin, M., \& Oliveira-Filho, A. (2009). Woody Plant Diversity, Evolution, and Ecology in the Tropics: Perspectives from Seasonally Dry Tropical Forests. Annual Review of Ecology, Evolution, and Systematics, 40(1), 437-457.

Plana, E. (2000). Introducción a la Ecología y Dinámica del Bosque Tropical. Curso sobre gestión y conservación de bosques tropicales. Centro Tecnológico Forestal de Cataluña. Recuperado de http:// www.bionica.info/biblioteca/Plana\%20Bach\%20 2000\%20Ecologia\%20bosque\%20tropical.pdf.

Quesada, M., Sánchez-Azofeifa, G.A., Álvaraez-Añorve, M., Stoner, K., Avila-Cabadilla, L., Calvo-Alvarado, J. C., Castillo, A., Esprírito-Santo, M., Fagundes, M., Fernandes, G. W., Gamon, J. A., Lopezaraiza-Mikel, M., Lawrence, D., Cerdeira, L. P., Powers, J. S., Neves, F., Rosas-Guerrero, V., Sayago, R., \& Sánchez-Montoya, G. (2009). Succession and management of tropical dry forests in the Americas: Review and new perspectives. Forest Ecology and Management, 258, 1014-1024.

Rangel-Ch, O., \& Velásquez, A. (1997). Métodos de estudio de la vegetación. En O. Rangel, P. D. Lowy \& M. Aguilar (eds.). Colombia diversidad biótica II. Tipos de vegetación en Colombia (pp. 59-82). Bogotá: Editorial Guadalupe Ltda.

Repizo, A., \& Devia, C. (2008). Árboles y arbustos del valle seco del río Magdalena y de la región Caribe colombiana: su ecología y usos-guía de campo. Pontífica Universidad Javeriana y Corporación Autónoma Regional del Canal del Dique (Cardique). Bogotá, Colombia. 332 p. 
Rodríguez, G., Banda-R, K., Reyes, S., \& Estupiñán, A. (2012). Lista comentada de las plantas vasculares de bosques secos prioritarios para la conservación en los departamentos de Atlántico y Bolívar (Caribe colombiano). Biota Colombiana, 13(2), 7-39.

Román, F. J., Levy, S., Aguirre, J. R., \& Sánchez, A. (2013). Árboles de la Selva Lacandona útiles para la restauración ecológica. Zapopan, Jalisco, México. Comisión Nacional Forestal, Coordinación General de Educación y Desarrollo Tecnológico, Gerencia de Desarrollo y Transferencia de Tecnología. 89 p.

Ruíz, J., Fandiño, M. C., \& Chazdon, R. L. (2005). Vegetation structure, composition and species richness across a 56-year chronosequence of dry tropical forest on Providencia Island, Colombia. Biotropica, 37(4), 397-407.

Ruíz, J., \& Fandiño, M. C. (2009). Estado del bosque seco tropical e importancia relativa de su flora leñosa, islas de la Vieja Providencia y Santa Catalina, Colombia, Caribe suroccidental. Revista Academia Colombiana de Ciencias, 33(126), 5-15.

Sánchez-Azofeifa, G. A., Quesada, M., Rodríguez, J. P., Nassar, J., Stoner, K., Castillo, A., Garvin, T., Zent, E., Calvo-Alcarado, J. C., Kalacska, M., Fajardo, L., Gamon, J. C, \& Cuevas-Reyes, P. (2005). Research Priorities for Neotropical Dry Forests. Biotropica, 37(4), 477-485

Torres, A. M., Adarve, J. B., Cárdenas, M., Vargas, J. A., Londoño, V., Rivera, k., Home, J., Duque, O. L \& González, A. M. (2012). Dinámica sucesional de un fragmento de bosque seco tropical del Valle del Cauca, Colombia. Biota Colombiana, 13(2), 66-85.

Uribe, A., Velásquez, P., \& Montoya, M. (2001). Ecología de poblaciones de Attalea butyracea (Arecaceae) en un área de bosque seco tropical (Las Brisas, Sucre, Colombia). Actualidades Biológicas, 23(74), 33-39.

Van Voss, O., Aguirre, N., \& Hofstede, R. (2001). Sistemas forestales integrales para la Sierra del Ecuador. Proyecto EcoPar-Universidad de Ámsterdam. Quito, Ecuador. 120 p.

Vargas, O. (2007). Guía metodológica para la restauración ecológica del Bosque Altoandino. Bogotá: Universidad Nacional de Colombia. Convenio Interinstitucional Acueducto de Bogotá -Jardín Botánico- Secretaría Distrital de Ambiente. 189 p.

Vargas, W., \& Ramírez, W. (2014). Lineamientos generales para la restauración del Bosque Seco Tropical en Colombia. En H. García \& C. Pizano (eds.). El bosque seco tropical en Colombia. (pp. 252-291). Instituto de Investigación de Recursos Biológicos Alexander von Humboldt $(\mathrm{IAvH})$. Bogotá: Ediprint Ltda.

Yepes, A., Del Valle, J., Jaramillo, S., \& Orrego, S. (2010). Recuperación estructural en bosques sucesionales andinos de Porce (Antioquia, Colombia). Revista de Biología Tropical, 58(1), 427-445.

Yepes, A., \& Villa, J. (2010). Sucesión vegetal luego de un proceso de restauración ecológica en un fragmento de bosque seco tropical (La Pintada, Antioquia). Lasallista de Investigación, 7(2), 24-34.

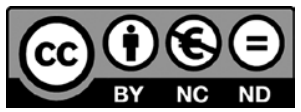

